\title{
REMOTE EDUCATION INTERVENTIONS FOR SYSTEMIC SCLEROSIS PATIENTS
}

Alessandra Rodrigues Hansen Ferreira ${ }^{1}$, Felipe Souza da Silva ${ }^{1}$, Luiza Castro Fernandes ${ }^{1}$, Thalles Guilherme de Almeida ${ }^{1}$, Barbara Niquini Assunção ${ }^{2}$, Caio Carvalhais Chaves ${ }^{1}$, João Victor de Pinho Costa ${ }^{1}$, Vivian Guerra de Faria ${ }^{1}$, Anderson Ramos Lisboa $^{1}$, Liliane Amaral ${ }^{1}$, Gilda Aparecida Ferreira ${ }^{1}$, Maria Raquel Costa Pinto ${ }^{1}$, Flávia Patrícia Sena Teixeira Santos ${ }^{1}$, Leandro Augusto Tanure ${ }^{1}$, Júnia Amorim Andrade ${ }^{1}$, Débora Cerqueira Calderaro ${ }^{1, *}$

1.Universidade Federal de Minas Gerais, Belo Horizonte (MG), Brazil; 2.Faculdade de Ciências Médicas de Minas Gerais, Belo Horizonte (MG), Brazil.

*Corresponding author: dccalderaro@gmail.com

\section{BACKGROUND}

Systemic sclerosis (SSc) is an autoimmune connective tissue disease characterized by excessive collagen deposition in the skin and internal organs with associated vasculopathy and autoantibody production, besides fibrosis and function loss. The function of the hands are usually impaired due to the development of Raynaud's phenomenon and fibrosis. To minimize these functional losses, in addition to drug treatment, rehabilitation and education measures are necessary. Educational interventions have been suggested as a good strategy to improve patients' awareness of their disease, leading to better self-management, autonomy and improved quality of life and function.

\section{METHODS}

As a consequence of the social distancing imposed by the COVID-19 pandemic, remote education interventions were designed. They were planned to occur through phone calls, e-mail, WhatsApp and other social media. Interventions include educational actions to provide information about SSc and the non-pharmacological measures to address signs and symptoms of the disease, besides measures for joint protection, energy conservation, selfcare, stretching and therapeutic exercises for the hands. Evaluation of patients' knowledge about their disease (through a standardized questionnaire developed by the researchers), quality of life (assessed by the descriptive system in three levels of five dimensions of EuroQol), depression (applying beck depression inventory) and function (using the Canadian occupational performance measure) will be performed before and after educational interventions and compared, in order to evaluate the impact on the groups.

\section{RESULTS}

Twenty-three SSc patients previously selected from the university hospital were contacted through phone calls. Four (17\%) were unreachable, $4(17 \%)$ were unwilling or unavailable to participate and $15(66 \%)$ showed great interest in the project. Out of these, $3(20 \%)$ individuals did not have access to e-mail or social media. Although it may impose an obstacle to adequately present all educational materials developed to provide information for the patients, including booklets, Instagram posts, images and videos, it will not prevent the awareness process. Patients willing to participate showed great enthusiasm and were receptive to the students performing the phone calls.

\section{CONCLUSION}

During the social distancing imposed to control the COVID-19 pandemic, many patients had their medical appointments postponed or canceled. Telemedicine and remote monitoring have become a reality in the management of these patients. The present work intends to provide education and guidance for SSc patients through periodic phone calls and distribution of online education materials developed for this proposal.

\section{KEYWORDS}

Systemic sclerosis, Patient education as topic, Health education. 